\title{
Unies pour le meilleur et pour le pire. Femmes africaines et villes coloniales : une histoire du métissage
}

Ch. Didier GONDOLA

\section{(2) OpenEdition}

Journals

Édition électronique

URL : https://journals.openedition.org/clio/377

DOI : 10.4000/clio.377

ISSN : 1777-5299

Éditeur

Belin

Édition imprimée

Date de publication : 1 novembre 1997

ISBN : 2-85816-346-4

ISSN : $1252-7017$

\section{Référence électronique}

Ch. Didier GONDOLA, « Unies pour le meilleur et pour le pire. Femmes africaines et villes coloniales une histoire du métissage », Clio. Histoire, femmes et sociétés [En ligne], 6 | 1997, mis en ligne le 01 janvier 2005, consulté le 23 avril 2022. URL : http://journals.openedition.org/clio/377 ; DOI : https:// doi.org/10.4000/clio.377

Ce document a été généré automatiquement le 23 avril 2022.

Tous droits réservés 


\title{
Unies pour le meilleur et pour le pire. Femmes africaines et villes coloniales : une histoire du métissage
}

\author{
Ch. Didier GONDOLA
}

\begin{abstract}
Une histoire des femmes qui serait celle de [leurs] victoires mconnues ou de [leurs] humiliations trop connues ne m'intresse pas vraiment. [...] Ce qui devient non seulement fascinant mais urgent, c'est de prendre le champ historique dans son entier, sans le restreindre au domaine fminin, en l'interrogeant autrement, faisant ressortir chaque fois qu'il est possible la division sexuelle des $\mathrm{r}^{\mathrm{TM}} l$ les. $\mathrm{C}^{\prime}$ est justement sur ce partage entre le masculin et le fminin que le silence de l'histoire s'est abusivement fait. (Arlette Farge, cite dans Perrot 1984 : 98).
\end{abstract}

1 L'histoire de la ville africaine, et notamment de la ville coloniale, continue à être articulée autour de plusieurs thématiques dominées par les hommes : les migrations, le travail, l'organisation sociale et les résistances politiques ${ }^{1}$. L'invisibilité des femmes dans cette histoire surprend, parce qu'il est connu aujourd'hui que, sans la présence des femmes, la ville coloniale n'aurait sans doute jamais acquis l'un de ses caractères les plus remarquables, le métissage culturel. De simples regroupements des services administratifs et points d'ancrage de l'effort colonial, à l'aube du XXe siècle, les villes coloniales sont devenues, au milieu des années 1950, des foyers intenses de diffusion de cultures métisses. Cette évolution s'est effectuée, dans la plupart des villes, grâce à l'arrivée des femmes qui, d'abord indésirables, furent progressivement autorisées à y résider dans les strictes limites d'une législation paternaliste. Au début de la colonisation, dans la plupart des villes récentes, les autorités coloniales prirent des mesures interdisant aux femmes de quitter le village pour venir en ville. Presque partout en Afrique, les femmes non-mariées restaient tenues en "otages $»^{2}$ dans les campagnes pour assurer le retour des hommes de la ville ${ }^{3}$. Autant dire que les femmes ont été exclues d'un domaine qui a été d'abord celui de l'Européen, et dans lequel les Africains eux-mêmes ont longtemps fait figure de " déracinés ", de "détribalisés »" et, finalement, d'«évolués». La fixation des villes coloniales, avec tout ce qu'elle a 
comporté d'érections symboliques (immeubles, monuments, routes, ponts, etc.) et d'échelles sociales et raciales, doit être regardée comme un acte éminemment masculin ${ }^{5}$. En face de ces structures verticales du pouvoir, dont elles se sont retrouvées le plus souvent bannies, les femmes ont opposé une série de lieux horizontaux, la rue, le marché, le bar, les fêtes, les associations d'entraide, qui ont été autant de carrefours de métissage. Si bâtir la ville coloniale a été l'affaire des hommes, la peupler n'a été également autorisé qu'aux seuls hommes : les Européens venant de la métropole et les Africains «sortant» de la "brousse». Dans un cas comme dans l'autre, et pour des raisons aussi bien économiques que sociales, la mobilité géographique n'a concerné que les hommes. Pour les ruraux africains, la migration vers la ville a symbolisé une sorte d'épreuve initiatique tandis que le mariage consacrait l'entrée des femmes dans l'âge adulte. Si cette situation a prévalu au début de la fixation des villes et pendant le premier quart du XXe siècle, elle change à la veille, et surtout au lendemain de la crise des années 1930. En dehors des femmes mariées qui viennent rejoindre leur mari, la ville devient ainsi le lieu où des femmes seules viennent tenter l'aventure citadine. Belinda Bozzoli a observé, dans une récente étude fondée sur un large corpus de témoignages oraux, les itinéraires des migrantes de Phoekeng vers Johannesburg. Comme pour les jeunes hommes, ces itinéraires ont symbolisé pour les jeunes femmes des étapes successives de prise de pouvoir et d'indépendance ${ }^{6}$. Dans un premier temps, dès les années 1920, il s'agit d'échapper aux contraintes de la société rurale ${ }^{7}$ puis, au cours de contacts ultérieurs avec la ville, d'accumuler meubles et trousseau en vue du mariage. Plus tard, lorsqu'elles sont bien installées en ville, les femmes de Phoekeng mettent de côté une somme qui, à la fin de leur séjour urbain, servira de rente et permettra l'achat d'une maison à Phoekeng'.

La guerre des sexes en ville coloniale

3 Si la ville coloniale fut «place de colonisation » et lieu du politique, donc du pouvoir9, ce fut aussi dans la négociation des identités et des fonctions sociales entre les hommes et les femmes. Partout où les citadines ont constitué une minorité numérique, cette négociation s'est déroulée dans des termes qui leur ont été bien souvent favorables. C'est aussi au travers de stratégies originales que les citadines ont défini leur zone d'influence, organisé leurs terrains d'initiative et même articulé une série de discours contestataires. De ce point de vue les hommes et les femmes ont eu une même ambition dont la migration en ville a constitué l'étape préliminaire. La différence tient dans le style et les moyens utilisés. Dans une situation où les hommes ont eu recours à une double légitimité coloniale et "coutumière », les femmes, doublement marginalisées, ont investi des domaines culturels à l'angle mort du contrôle colonial et en rupture avec les lois « coutumières » pour se rendre visibles et accroître leur pouvoir social en ville. Ce pouvoir s'est d'autant mieux affermi que les femmes africaines ont évité une confrontation directe avec les hommes, s'effaçant en public devant leur pouvoir social pour mieux les dominer en privé ${ }^{10}$. Une observation similaire est effectuée par Susan Rogers dans son article sur le pouvoir des hommes et des femmes dans le monde paysan. Le mythe de la domination des hommes fonctionne, selon Rogers, dans l'intérêt des paysannes et des paysans, en donnant l'apparence (appearance) du pouvoir aux derniers et la réalité du pouvoir (actual power) aux premières.

Women [...] buy their power by granting men autorithy and respect, assuming that if they allow men to believe that male dominance actually exists, men will not notice that women are actually wielding a considerable amount of power. ${ }^{11}$ 

guerre des sexes dans les domaines (le travail salarié13, l'éducation scolaire, l'administration et la politique) que la société coloniale avait d'office réservés aux hommes. Au contraire, les femmes ont exploré des domaines que l'on suppose généralement absents de tout enjeu de pouvoir et où, cependant, s'élaborent ce que James Scott, dans son étude sur les paysans malais, appelle « hidden transcripts ${ }^{14}$, le pouvoir des faibles, pouvoir social dont l'influence sur le changement des structures du quotidien est cardinal. contrairement au milieu rural, elles se sont distinguées par leur mobilité. Attachées à la terre par les différents cycles agraires, les femmes, une fois en ville, avaient tout loisir d'effectuer des séjours réguliers au village tandis que les hommes se retrouvaient mobilisés par les contraintes du travail salarié. La ville a également permis aux femmes de monnayer leurs charmes et de négocier des unions matrimoniales non plus seulement, comme au village, en fonction de l'appartenance ethnique du prétendant, mais en faisant valoir des critères tels que l'éducation, l'âge, le statut social et, fait nouveau, l'amour ${ }^{15}$. Enfin, comme l'ont établi les premières études urbaines, la ville coloniale fut, en fait, le seul lieu où les femmes ont pu devenir propriétaires ${ }^{16}$, grâce au commerce, au petit artisanat, à la domesticité ${ }^{17}$, sans oublier le rôle économique de la prostitution ${ }^{18}$ qui a constitué une source principale d'accumulation pour les premières citadines. A Nairobi, selon les informations recueillies par Luise White, c'est à l'aide du revenu accumulé grâce à la vente des services domestiques et sexuels que certaines femmes se sont bâties de grosses fortunes immobilières et sont devenues, comme le déclare une informatrice, des «femmes puissantes ${ }^{19}{ }^{1}$. Ces « semi-prostituées ${ }^{20}$ » qui au centre extra-coutumier de Kisangani regroupaient, en 1944, un effectif de 2093 femmes (soit la moitié du nombre des femmes mariées) ont exercé une ponction financière considérable sur une section de la population « évoluée » et sur certains Européens, en échange de services sexuels variés. Sous ses différentes variantes, la prostitution s'est révélée pour les citadines africaines un moyen efficace de sélection sexuelle, de mobilité sociale, notamment grâce à l'hypergamie ${ }^{21}$, et de mobilité spatiale, dans le cas de la prostitution itinérante ${ }^{22}$.

6 Bien qu'il convienne de reconnaître que ce fut par le biais de la prostitution que les citadines ont pu déjouer les handicaps de la ville coloniale, c'est pourtant grâce au commerce qu'elles ont gagné leur indépendance économique vis-à-vis des hommes. Dans les villes d'Afrique de l'ouest, les femmes se sont surtout imposées dans les marchés, avec leurs réseaux d'affaires dominés par les market queens ${ }^{23}$, tandis qu'en Afrique centrale, orientale et australe, les cas rencontrés concernent davantage l'artisanat, le colportage et le commerce à domicile, mais comportent autant de success stories.

7 Face à cette réussite économique des femmes, la perception et les attitudes des autorités coloniales se sont construites autour de plusieurs réponses ambiguës. L'opinion selon laquelle la citadine africaine demeure " a liability rather than an asset $»^{24}$, formule que l'on doit au commissaire de police de Dar-es-Salaam, résume assez bien la position coloniale. Tant que les femmes restaient économiquement dépendantes des hommes, leur résidence en ville était tolérée, même pour celles qui venaient s'y livrer à la prostitution. Dès que les femmes ont commencé à se soustraire à cette dépendance, 
les mesures prises par les municipalités ont tendu à contrôler, voire ruiner les sources de leur émancipation économique ${ }^{25}$.

Pour les hommes africains, la volonté de contrôler le pouvoir économique de leurs femmes fut tout autant ambiguë. En apparence, l'enjeu économique prime ${ }^{26}$. Les hommes ont tenu à contrôler le revenu drainé par leurs femmes parce qu'ils se sont vite aperçus qu'en ville coloniale pouvoir économique signifiait d'abord émancipation sociale.

If my wife earns money she becomes independent and does what she likes. I cannot control her ${ }^{27}$.

9 En réalité, il est surtout question d'une négociation des rôles et des identités sociaux dans laquelle la querelle financière sert d'alibi ${ }^{28}$.

My wife is not to work because I cannot get any suitable... light work that would not ruin her health and thus ruin the chances of expected offsprings ${ }^{29}$.

My wife drinks, both kaffir beer and brandy, and I think it is because she is employed ${ }^{30}$.

Il s'est moins agi, de la part des hommes, de limiter le pouvoir économique des femmes que de contrôler leur identité, leur mobilité et leur visibilité; de leur assigner des fonctions sociales figées et, en même temps, de protéger leurs propres prérogatives dans le cadre d'une société mouvante, la ville coloniale, où les hommes n'étaient plus à l'abri du célibat ni du chômage et devaient se plier aux lois de la monogamie.

11 Femmes africaines en ville coloniale : le cas de Kinshasa

12 Cette perception de la femme a prévalu dans une ville comme Kinshasa, où les Belges avaient longtemps cultivé le rêve d'une société parfaite, débarrassée de la polygamie, du célibat, du chômage, des femmes libres et des migrants flottants; une société urbaine faite de couples monogames, d'ouvriers dociles et de ménagères soumises. La réalité manifesta, par contraste, une société urbaine émaillée de tensions et dominée, dans tous ses secteurs, par des contradictions et des rapports de forces permanents.

L'arrivée des premiers éléments féminins, dans ce qui n'était alors qu'un immense camp de travailleurs, coïncide avec la baisse des activités économiques à Kinshasa, en 1921-22 $2^{31}$, et surtout avec la crise des années 1930, durant laquelle des milliers de travailleurs touchés par le chômage furent rapatriés manu militari vers les zones rurales $^{32}$. Ces premières migrantes étaient composées de femmes indépendantes qui profitèrent de la crise pour se positionner en ville aux marges de l'économie formelle. En 1928 déjà, elles étaient au moins 5000 femmes, non mariées, dont la majorité était engagée dans la culture maraîchère dans les zones vertes de la périphérie de Kinshasa et à l'intérieur de la barrière écologique séparant la ville blanche des cités indigènes. Un petit groupe d'entre elles s'était spécialisé dans la fabrication et la vente de charbon, d'huile de palme, de poisson et de pain de manioc. D'autres comptaient exclusivement sur la prostitution comme moyen de subsistance en ville. En face de ces indépendantes, la catégorie des femmes mariées (au nombre de 358 seulement en 1928) a constitué la minorité silencieuse et invisible, confinée dans les soins maternels et les travaux domestiques, protégée, à l'ombre du mari monogame et "évolué ", des bouleversements culturels dont la ville coloniale était le théâtre.

14 L'une des premières mesures affectant directement le statut économique des femmes indépendantes fut, en 1922, l'article 4 du nouveau " contrat de travail entre indigènes et maîtres civilisés " réglementant le travail des femmes. Désormais, seule la femme mariée « civilement, religieusement ou suivant la coutume » était autorisée à « engager 
ses services sur l'autorisation expresse ou tacite de son mari ${ }^{33}$ ». En 1929, convaincue par l'idée que l'immense majorité des citadines non mariées vivait de la prostitution, la municipalité de Kinshasa ordonna aux «femmes indigènes adultes et valides vivant théoriquement seules » de payer une taxe annuelle de 50 francs $^{34}$, l'équivalent de dix journées de travail d'un boy d'Européen, la catégorie la mieux payée à l'époque. Outre l'accès au marché du travail urbain ${ }^{35}$, la marginalisation des femmes se banalisa dans d'autres domaines, l'éducation et les loisirs et, plus tard, la politique. La terminologie coloniale suggère d'ailleurs une série d'oppositions dans la socialisation des femmes et des hommes en ville coloniale. Le français est réservé au citadin tandis que des termes lingala stigmatisent la citadine. On parle, de manière laudative, d'« évolué " mais de musenzi (« paysanne »), d'« immatriculé » ${ }^{36}$ mais de ndumba (prostituée). En revanche, le terme « indigène ", qui a acquis au fil du temps une connotation péjorative, continue à désigner les femmes en ville, mais « évoluant "s'applique désormais aux migrants des années 1950. Derrière cette labellisation se profile une dichotomie des sexes dont les termes -culture/nature, modernité/tradition, ville/campagne, public/privé- opposent l'homme et la femme. Civilisé et cultivé, le citadin africain était supposé, à son tour, guider sa compagne vers cette aventure évolutionniste ; vision idéale qui s'est traduite, dans la réalité, par ce que le colonisateur évoque comme «le drame des ménages d'évolués où un mari qui a pris conscience de toute sa personnalité de civilisé... doit passer sa vie à côté d'une mosenji ${ }^{37}$. " Du côté des évolués eux-mêmes, "évolution » et " éducation » ont un sens différent selon les sexes :

La femme d'un volu, sa vie tant calque sur celle de son mari, passe aussi pour volue.

Mais elle ne l'est pas en ralit. Cette lacune tant remarque, la cration des foyers sociaux dans tous les grands centres se fit. Des assistantes sociales s'y consacrent ^ donner le meilleur d'elles-mmes aux pauvres femmes congolaises ${ }^{38}$.

15 Pour l'évolué, les aptitudes de la femme éduquée et évoluée concernent avant tout la sphère domestique et privée : bien tenir sa maison, bien cuisiner, savoir vivre et éviter les commérages. On lui recommande donc de "visiter des maisons de ménage européen... ce genre de visites, réparties sur un bon nombre de mois, auront certainement une emprise favorable ${ }^{39}$. "

Cette identité sociale, un groupe de femmes l'ont refusée. Les femmes libres de Kinshasa ont choisi, au contraire, de se définir autrement et de façonner leurs identités sociales aux carrefours du métissage. Dans une ville où la plupart des citadins se sont d'abord socialisés en fonction de l'appartenance ethnique et ont fini par «tribaliser » le politique, les femmes ont été les premières ${ }^{40}$ à s'organiser sans référence aux critères ethniques ${ }^{41}$ et à créer des solidarités nouvelles fondées sur l'entraide et les activités récréatives et qui ont, à leur façon, cristallisé un discours politique ${ }^{42}$. Ces femmes se sont rendues visibles en ville, dans le cadre des bars-dancings, à travers deux véhicules identitaires inséparables: les associations d'élégance et la musique. Actrices incontournables dans le jeu nocturne qui se mettait en place dans les bars de la capitale, les femmes libres ont redistribué les rôles sociaux en imposant leurs propres règles aux évolués qui s'y pressaient. On a assisté à un véritable renversement des rôles dû en grande partie au fait que les femmes, démographiquement en minorité, ont monnayé leur charme et, pour la première fois, ont eu recours à la polyandrie pour asseoir leur nouvelle autorité sur les hommes. Celles qu'on appelait bana (les enfants, c'est-à-dire les jeunes filles) s'étaient entourées d'une cour de prétendants tandis que les mama mikonzi (mères-chefs) ou basi ya kilo ${ }^{43}$ (femmes déjà plus âgées) régnaient sur des hommes beaucoup plus jeunes, mais tout aussi émasculés. Les associations 
d'élégance ont constitué le cadre par excellence de cette prise de pouvoir par les femmes. Là se rencontraient des femmes jeunes, célibataires, en rupture avec le cadre «traditionnel », auxquelles des mama mikonzi-qui, elles, avaient déjà « fait leur vie » et s'étaient amassées un petit pécule après plusieurs liaisons avec des Européens ou des évolués-assuraient l'éducation. Lors de leurs réunions dans les bars de la capitale, les bana exhibaient une mode métisse ${ }^{44}$ faite de pagnes luxueux, aux coloris chatoyants, de bijoux voyants et de sacs à main directement importés de métropole. En revanche, au lieu du rouge à lèvre, elles jaunissaient leurs lèvres à l'aide d'une plante locale appelée nzete ya mino, refusaient le port de la jupe et de la robe ${ }^{45}$, parlaient exclusivement lingala et rejetaient la cuisine européenne au profit de plats épicés locaux, même si une grande majorité ne savait pas faire la cuisine.

De cette visibilité, à l'aide de hidden transcripts, à l'affirmation de leur pouvoir social et économique, il n'y avait qu'un pas, rapidement franchi par les femmes de Kinshasa. Elles détiennent aujourd'hui des positions stratégiques dans la «deuxième économie ${ }^{46} »$, gagnent parfois jusqu'à trois fois plus que leurs maris ${ }^{47}$ et contribuent financièrement à l'éducation de leurs enfants ${ }^{48}$. Grâce à leur mobilité-qui les amènent en Europe, en Amérique et jusqu'à Hong-Kong-, elles ont su, mieux que les hommes, faire face à la crise économique et se retrouvent aux commandes d'entreprises florissantes. Cette réussite économique permet aujourd'hui à certaines de ces femmes d'exercer une réelle domination sur les hommes ${ }^{49}$.

Pour une histoire du métissage

19 Le cas de Kinshasa montre qu'il faut se garder de « constituer un nouveau territoire qui serait l'histoire des femmes, tranquille concession où elles se déployeraient à l'aise, à l'abri de toute contradiction; mais bien davantage de changer la direction du regard historique, en posant la question du rapport des sexes comme centrale. L'histoire des femmes, en somme, n'est possible qu'à ce prix ${ }^{50}$."

La ville coloniale a donc propulsé les femmes africaines dans de nouveaux rôles sociaux qui figurent parmi les bouleversements majeurs qui affectent les villes africaines. De la rencontre entre les femmes africaines et la ville coloniale les femmes ont gagné en visibilité et surtout en autonomie; la ville coloniale a acquis une fonction de creuset culturel. La mode, la musique, la danse, la fête, la peinture, l'humour populaire, les syncrétismes religieux et les fabrications argotiques représentent autant de cultures métisses nées de cette rencontre et dont il importe aujourd'hui d'explorer les développements. C'est dans ces domaines métis, qui sont loin d'être purement récréatifs, que les identités sociales des hommes et des femmes sont mises en question, négociées et redéfinies.

21 Si les domaines traditionnels de l'histoire nous ont habitués à des rôles tranchés et définitifs, dans ces domaines métis, la féminité et la masculinité se présentent comme des rôles interchangeables, en permutation permanente et résultats de jeux de forces intenses. Un exemple, parmi d'autres, est celui de la musique zaïroise dont l'étude non seulement permet de représenter autrement les rôles des femmes et des hommes dans l'histoire, mais également de revisiter le politique ${ }^{51}$. Si le politique se résume aux échéances électorales, aux débats politiques et aux réformes institutionnelles, alors, d'une part, en sont exclues les femmes et, d'autre part, l'histoire politique moderne du Zaïre commence avec la formation des premiers partis politiques, en 1956, et les élections communales de 1957. Si, en revanche, on examine de près le pouvoir que les femmes se sont attribuées à travers les cultures populaires, il est évident que le 
politique devient un champ d'étude plus large dans lequel la contribution des femmes, et aussi des jeunes, a sa place.

\section{BIBLIOGRAPHIE}

AULT, James, 1983, « Making 'Modern' Marriage `Traditional' : State Power and the Regulation of Marriage in Colonial Zambia », Theory and Society, 12, 181-210.

BALANDIER, Georges, [1955] 1985, Sociologie des Brazzavilles noires, Paris : Fondation Nationale des Sciences Politiques.

BERGER, Iris, 1992, Threads of Solidarity. Women in South African Industry, 1900-1980, Bloomington : Indiana University Press.

BOZZOLI, Belinda (with the assistance of Mmantho Nkotse), 1991, Women of Phoekeng. Consciousness, Life Strategy, and Migrancy in South Africa, 1900-1983, Portsmouth, NH : Heinemann.

CUTRUFELLI, Maria Rosa, 1983, Women of Africa. Roots of Oppression (traduit de l'italien par Nicolas Romano), London : Zed Press.

ENYUKA NGAMBA, 1988, Le secteur informel et la création d'emplois à Kinshasa, Kinshasa : Université de Kinshasa.

GONDOLA, Ch. Didier, 1997, « Oh, rio-Ma! Musique et guerre des sexes à Kinshasa, 1930-1990 », Revue française d'histoire d'outre-mer, t. 84, n 314, pp. 51-81.

- 1996a, Villes miroirs : migration et identités urbaines à Brazzaville et Kinshasa, 1930-1970, Paris :

L'Harmattan.

- 1996b, «Popular Music, Urban Society, and Changing Gender Relations in Kinshasa, Zaire », in Grosz-Ngaté, Maria \& Omari H. Kokole (eds), Gendered Encounters: Challenging Cultural Boundaries and Social Hierarchies in Africa, pp. 65-84, London \& New York : Routledge.

- 1992, «Ata ndele... et l'indépendance vint. Musique, jeunes et contestation politique dans les capitales congolaises ", in Coquery-Vidrovitch, Catherine \& alii (eds), Les jeunes en Afrique. La politique et la ville, pp. 463-487, tome 2, Paris : l'Harmattan.

GRUNDFEST, Schoepf \& Walu ENGUNDU, 1991, «Women's Trade Contributions to Household Budgets in Kinshasa ", in MacGaffey, Janet (ed), The Real Economy of Zaire. The Contribution of Smuggling and Other Unofficial Activities to National Wealth, pp. 124-151, Philadelphia : University of Pennsylvania Press.

GUGLER, Josef \& William FLANAGAN, 1978, Urbanization and Social Change in West Africa, Cambridge : Cambridge University Press.

HANSEN, Karen, 1989, Distant Companions. Servants and Employers in Zambia, 1900-1985, Ithaca : Cornell University Press.

HUNT, Nancy Rose, 1991, « Noise over Camouflaged Polygamy, Colonial Morality Taxation, and a Woman-Naming Crisis in Belgian Africa », Journal of African Studies 32, 471-494. 
JACOBS, Susie, 1995, « Gender Divisions and the Formation of Ethnicities in Zimbabwe », in Stalsiulis, Daiva \& Nira Yuval-Davis (eds), Unsettling Societies : Articulations of Gender, Race, Ethnicity and Class, pp. 241-263, London : Sage Publications.

KNIBIEHLER, Yvonne \& Régine GOUTALIER, 1985, La femme au temps des colonies, Paris : Stock.

LITTLE, Kenneth, 1973, African Women in Towns. An Aspect of Africa's Social Revolution, Cambridge : Cambridge University Press.

- 1974, Urbanization as a social process. An essay on movement and change in contemporary Africa, London \& Boston : Routledge \& Kegan Paul.

MACGAFFEY, Janet, 1986, « Women and Class Formation in a Dependent Economy : Kisangani Entrepreneurs ", in Robertson, Claire \& Iris Berger, Women and Class in Africa, pp. 161-177, New York : Africana Publishing Company.

MATONDO KUBU, Turé, 1988, « Amour et permutation ludique des rôles sociaux entre l'homme et la femme dans la chanson contemporaine des orchestres congolo-zaïrois ", Cahiers congolais de l'imaginaire 2, juillet, 20-24.

MAYER, Philip \& Iona, 1974, Townsmen or Tribesmen. Conservation and the Process of Urbanization in a South African City, Capetown : Oxford University Press.

MBILINYI, Marjorie, 1989, « 'This Is an Unforgettable Business' : Colonial State Intervention in Urban Tanzania ", in Parpart, Jane \& Kathleen Staudt (éds), Women and the State in Africa, pp. 111-129, Boulder \& London : Lynne Rienner Publishers.

OBBO, Christine, 1980, African Women : Their Struggle for Economic Independence, London : Zed Press.

PARPART, Jane, 1986, « Class and Gender on the Copperbelt : Women in Northern Rhodesian Copper Mining Communities, 1926-1964», in Robertson, Claire \& Iris Berger (eds), Women and Class in Africa, pp. 141-160, New York : Africana Publishing Company.

PERROT, Michelle (dir.), 1984, Une histoire des femmes est-elle possible ? Marseille : Rivages.

ROGERS, Susan Carol, 1975, «Female Forms of Power and the Myth of Male Dominance : A Model of Female/Male Interaction in Peasant Society ", American Ethnologist 2 (4), November, pp. 727-56. SCOTT, James, 1990, Domination and the Arts of Resistance, New Haven \& London : Yale University Press.

VIDAL, Claudine, 1991, Sociologie des passions (Côte-d'Ivoire, Rwanda), Paris : Karthala.

WHITE, E. Frances, 1991, Sierra Leone's Settler Women Trader : Women on the Afro-European Frontier, Ann Arbor : University of Michigan Press.

WHITE, Luise, 1990, The Comforts of Home. Prostitution in Colonial Nairobi, Chicago : University of Chicago Press.

\section{NOTES}

1. La rubrique « femmes » (la rubrique « hommes » n'apparaît jamais) dans les index des différents ouvrages consacrés à la ville africaine est une bonne indication de cette volonté de faire figurer les femmes dans une histoire dont les hommes sont implicitement tenus pour les seuls vrais acteurs. Ce qui me paraît plus aberrant encore est de retrouver cette rubrique «femmes » dans les index d'ouvrages pourtant dévolus aux femmes. 
2. Little $1973: 18-19$, Cutrufelli $1983: 22$.

3. Dans le Copperbelt zambien, les chauffeurs de camions de transport de personnes qui étaient surpris embarquant ou débarquant des femmes encouraient le risque de se faire arrêter. La plupart des conducteurs ignoraient ces prescriptions et se laissaient corrompre par les femmes qui montaient et descendaient entre les arrêts réglementaires, évitant ainsi les contrôles intempestifs (Ault 1983 : 183, Parpart 1986 : 149). Un cas extrême est celui de Kampala où, dans les années 1950, les autorités municipales adoptèrent une série de décrets visant à l'expulsion de toutes les femmes non-mariées surprises « traînant » (loitering) en ville (Obbo 1980 : 26).

4. La ville coloniale selon P. Naville, cité dans Balandier (1955:114), est « l'agglomération de masses humaines déracinées, qui campent dans l'attente d'un travail rémunérateur, et qui pourtant conservent des liens... avec la brousse natale ». 5. Knibiehler \& Goutalier (1985:17) ont insisté sur la nature virile de l'acte colonisateur, écrivant que « coloniser est un acte essentiellement masculin, c'est conquérir, pénétrer, posséder, féconder... »C'est, à mon avis, en ville coloniale que cette volonté de puissance s'est manifestée de la manière la plus intense et la plus fertile. Je crois aussi qu'il y a place pour une variété d'interprétations symboliques de cet acte que Knibiehler \& Goutalier ont abusivement réduit à sa seule dimension « romantique ».

6. Bozzoli 1991 : 91 ; voir également Obbo 1983 : 144, Mayer $1974: 248$ et Balandier $1955: 192$.

7. Avec la colonisation, les paysannes ont parfois perdu le droit de propriété et d'accumulation en milieu rural ; voir Jacobs (1995 : 247) pour le cas zimbabwéen.

8. Bozzoli 1991 : 81, 93, 106.

9. Sans prétendre fournir une définition du pouvoir, on peut dégager une distinction entre pouvoir et autorité, cette dernière étant la forme du pouvoir ritualisé, symbolisé, hiérarchisé et légitimé. Dans l'histoire, les femmes ont été peu souvent investies d'autorité, tandis qu'au contraire elles ont eu tendance à concentrer un pouvoir dont la nature et l'exercice restent souvent insaisissables ; voir Rogers 1975.

10. Obbo $(1980: 102,104,144)$ insiste sur l'usage de la duplicité (deceit), de la ruse (cunning) et de la « soumission traditionnelle » par les citadines africaines dans leur quête de pouvoir, de richesse et de statut social.

11. Rogers $1975: 748$. Traduction : « Les femmes [...] achètent leur pouvoir en accordant aux hommes autorité et respect ; elles supposent que si elles leur permettent de croire à la domination masculine, les hommes ne remarquent pas que les femmes détiennent beaucoup de pouvoir ».

12. Voir, pour exemples, le rôle politique des commerçantes ghanéennes qui, en 1968-69, ont obtenu une représentation politique à l'assemblée constituante (Gugler \& Flanagan $1978: 140$ ) ; la participation des femmes dans les grèves des années 1940-50 qui ont paralysé la production minière du copperbelt zambien (Parpart 1986 : 148-49) ; la révolte des femmes Ibo, au Nigéria, dans les années 1920, contre un décret de taxation par le gouvernement colonial (Little 1973 : 61, White $1987: 107$ ) et, dans les années 1950, celle des femmes swahili à Bujumbura (Hunt 1991 : 471).

13. A Kisangani, $2 \%$ des femmes, contre $90 \%$ des hommes, étaient engagées dans des activités salariales en 1952 (Pons $1969: 52$ ).

14. Scott 1990 : xii, 136.

15. La rédaction de lettres d'amour touchantes, par les prétendants, est un exemple des nouveaux critères de sélection prisés par les femmes, voir Bozzoli 1991 : 112-113. 
16. Little $1973: 21$ et Mayer $1974: 234$.

17. Dominée par les hommes (en 1948, les hommes constituaient $86 \%$ de la domesticité au Zimbabwe ; Jacobs 1995 : 248), la domesticité pour les femmes, en situation coloniale, a rarement existé sans son versant sexuel ; voir Knibiehler \& Goutalier 1985, Hansen 1989.

18. Elle recouvre plusieurs stratégies qui vont de la prostitution occasionnelle, source de revenus d'appoint, limitée au temps de la rencontre, au « concubinage relaché », en passant par des formes prostitutionnelles plus lucratives mais aussi plus intensives. 19. White $1990: 88,119$.

20. Pons $1969: 215$.

21. Voir Parpart 1986 : 153-154. Dans le copperbelt zambien cette pratique avait pris le nom de « mine marriage », une stratégie utilisée par les femmes pour changer de partenaires et rester en ville. Ault (1983) démontre comment, à l'aide d'une juridiction moderne, les tribunaux municipaux, le « mine marriage » a été finalement «traditionnalisé. »

22. A Kinshasa, au début des années 1920, certaines femmes quittaient la ville pour aller offrir leurs services aux Européens débarquant à Thysville, Matadi et même Boma (L'avenir colonial belge, 6 mars 1921, p. 7). A Naïrobi, devant l'insolvabilité de la clientèle africaine, et pour se soustraire à la multiplication des services extra-sexuels exigés, les « wazi-wazi » ciblèrent la clientèle européenne dans les années 1947-50 (White 1990 : $160,162,183)$.

23. A noter l'exemple remarquable de Freetown où, dès 1850 , les femmes ont démontré leur pouvoir économique en persuadant l'administration coloniale de construire le « Grand Marché » (White $1987: 108$ ).

24. Cité dans Mbilinyi $1989: 115$.

25. Voir les tensions soulevées par la production de bière locale à Dar-es-Salaam, dans les années 1930 (Mbilinyi 1989 : 121). Dans les villes minières zambiennes, malgré l'interdiction de la production artisanale de bière, renforcée par des amendes et des arrestations, les femmes poursuivirent cette activité lucrative (Parpart 1986 : 144,150). A Johannesburg, les productrices furent victimes de raids brutaux de la part des policiers (Berger 1992 : 24, 68).

26. Dans un contexte économique de crise, comme ce fut le cas à Matadi dans les années 1930, certains hommes sont allés jusqu'à encourager leurs femmes à se livrer à la prostitution tout en s'assurant la mainmise sur les sommes recueillies ; lire Gondola 1996a : 90.

27. Mayer $1974: 212$. Traduction : «Si ma femme gagne de l'argent, elle devient indépendante et fait ce qu'elle veut. Je ne peux la contrôler ».

28. Lire les remarques provocantes de Vidal 1991: 37 dans le cadre abidjanais des années 1980.

29. Mayer $1974: 212$. Traduction : «Ma femme n'est pas au travail parce que je ne peux pas lui trouver d'activité qui ne ruine pas sa santé et ses chances de mettre au monde une descendance souhaitée ».

30. Ibid. Traduction : «Ma femme boit à la fois de la bière et de l'eau de vie, et je pense que c'est parce qu'elle travaille ».

31. Un quart des HAV (hommes adultes valides) s'était retrouvé sans emploi ; Le progrès colonial, 13 avril 1922, repris dans Le courrier d'Afrique, 30 juillet 1932, p. 7.

32. Ville à vocation industrielle, Kinshasa a connu, entre 1929 et 1935, une perte de moitié de sa population masculine contre un gain de $8 \%$ de la population féminine. 
33. Bulletin officiel du Congo belge, 1922, p. 355.

34. Bulletin administratif du Congo belge, 1931, p. 11. En 1946, l'enquête d'Emmanuel Capelle sur Kinshasa indiquait que 11,5\% de la population féminine, soit 5000 femmes, payaient la taxe urbaine ; citée dans Balandier 1955 : 144.

35. C'est seulement en 1955 que des travailleuses furent engagées pour la première fois dans une usine de Kinshasa. L'expérience avorta lorsque l'Utexléo décida, la même année, de remplacer par des hommes toutes les femmes qu'elle avait embauchées dans ses unités de filature.

36. C'est-à-dire détenteur de la carte du mérite civique ; voir Gondola 1996a : 217.

37. Mission pédagogique Coulon-Deheyn-Renson, La réforme de l'enseignement au Congo belge, Congo belge, ministère des Colonies, conseil supérieur de l'Enseignement, décembre 1954, p. 235.

38. La voix du Congolais, janvier 1954, p. 19.

39. Ibid.

40. À l'exception des boys d'Européens qui s'étaient constitués en « association des boys de Léopoldville » aux lendemains de la crise des années 1930. Mais, même ici, les solidarités ethniques ont prévalu puisque la grande majorité des boys de Kinshasa étaient des Bayaka ressortissants du Kwango.

41. Elles ont également suscité l'association des métis, autorisée par le district urbain de Léopoldville à la fin de 1933 et qui a surtout servi de foyer aux enfants métis abandonnés (191 en 1934).

42. Gondola $1996 \mathrm{~b}$ : 76.

43. Littéralement " femmes de poids »; expression qui non seulement se réfère à leur statut social, mais souligne également leur imposante stature. C'est l'équivalent des nana Benz des villes côtières de l'Afrique de l'ouest.

44. Comparée à la construction du paraître féminin, la mode masculine s'est davantage caractérisée par le mimétisme et aussi une forme d'aliénation culturelle ; lire Gondola $1997: 64-66$.

45. «À une époque où les femmes en pagnes, considérées comme `sauvages' par rapport aux 'évoluées' en robe, n'avaient pas accès à certains magasins, hôtels ou pâtisseries en ville, les ndoumba se sont toujours refusées au port de la robe »; Aimée Mambou Gnali, «La Ndoumba congolaise », Etumba, 28 septembre 1967.

46. En 1984, à Kinshasa, 116 sur 200 « indépendants » étaient des femmes ; voir Enyuka Ngamba $1988: 7$.

47. Schoepf \& Engundu $1991: 127$.

48. Ibid., p. 131.

49. Dans sa chanson Mario, le musicien zaïrois Luambo Makiadi décrit les frasques amoureuses d'une quinquagénaire, riche commerçante, et de son jeune amant, Mario ; lire mon analyse de cette chanson (Gondola 1996b ; 1997) et le superbe, mais court, article de Matondo Kubu (1988).

50. Perrot $1984: 15$.

51. D'une manière inattendue, les premiers prisonniers politiques zaïrois ne sont pas des leaders politiques mais un " prophète », Simon Kimbangu, condamné à mort en 1923 pour avoir prédit l'indépendance en 1960, et un musicien, Adou Elenga, arrêté en 1954 pour avoir chanté Ata ndele (Gondola 1992). 


\section{RÉSUMÉS}

Une histoire méconnue ? Celle des femmes africaines, celle de la ville coloniale, ou plutôt celle du métissage qui a présidé à la rencontre des femmes et des cultures urbaines. Une histoire que l'on ne comprend qu'en prenant la mesure du rôle phare des cultures populaires que les femmes ont investi dès que les lois coloniales leur permirent le droit de cité à Kinshasa, à l'origine un campement pour travailleurs célibataires que l'arrivée des femmes a transformé en espace social métis. Les femmes ont fait la ville (coloniale), façonné de nouveaux styles de vie et permis aux citadins africains de prendre en charge, via la musique, la fête, le mouvement associatif, le petit commerce, un domaine qui avait été conçu par les autorités coloniales comme un laboratoire et un instrument de contrôle social, et aussi politique.

Widely over looked? underinvestigated ? Treated separately, the history of colonial Kinshasa and that of African women have been thoroughly researched. We are hard pressed to find these two histories intertwined, however. The history of both colonial Kinshasa and African women can only be fully understood by reffering to the critical role of popular cultures invested by women, when colonial laws permitted them to reside in the city. Due to the migration of women Kinshasa was transformed into a unique social milieu. Women revamped the city and created new lifestyles. They helped African city-dwellers reclaim the usage of the urban milieu via popular music, festive culture, associative movement, and petty trade in order to undermine the social and political control exercised by colonial authorities.

\section{AUTEUR}

\section{CH. DIDIER GONDOLA}

Ch. Didier GONDOLA a obtenu un doctorat en histoire à l'université Paris 7 (1993) et enseigne l'histoire au département d'histoire de Macalester College (Minnesota). Il est l'auteur de Villes miroirs : migrations et identités urbaines à Kinshasa et Brazzaville (1997), paru chez l'Harmattan, et d'une douzaine d'articles sur les cultures populaires, notamment la musique, en Afrique centrale. 